\title{
Erratum: Microscopic coupled-channels calculations of nucleus-nucleus scattering including chiral three-nucleon-force effects [Phys. Rev. C 93, 014607 (2016)]
}

\author{
Kosho Minomo, Michio Kohno, and Kazuyuki Ogata \\ (Received 6 June 2017; published 27 November 2017)
}

DOI: 10.1103/PhysRevC.96.059906

As reported in the Erratum [1], several errors were found in the program code for the nuclear-matter calculation reported in Ref. [2]. Changes in the $G$ matrices in nuclear matter including the three-nucleon force (3NF) effects affect the results of subsequent applications of them to the description of nucleon-nucleus and nucleus-nucleus scatterings reported in the original paper. We present the corrected results in this Erratum.

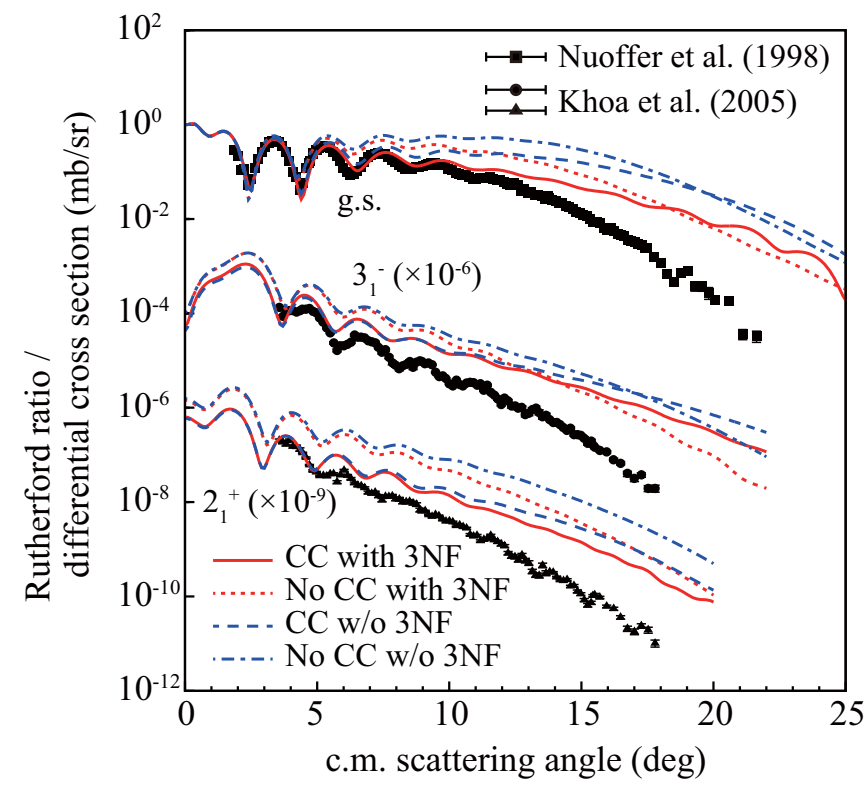

FIG. 1. Revised version of Fig. 1(a) in the original paper. Differential cross sections of elastic and inelastic ${ }^{16} \mathrm{O}-{ }^{16} \mathrm{O}$ scatterings at $70 \mathrm{MeV} /$ nucleon. The solid (dashed) line corresponds to the result with (without) the $3 \mathrm{NF}$ effects, and the dotted (dot-dashed) line corresponds to the result of the one-step calculation with (without) the $3 \mathrm{NF}$ effects. The experimental data are taken from Refs. $[3,4]$. The inelastic cross sections are scaled by the factor shown inside the panel for clarity.
The 3NF effects become weaker except for the real part in the ${ }^{3} \mathrm{O}$ channel. The corrected angular distributions of the ${ }^{16} \mathrm{O}-{ }^{16} \mathrm{O}$ and ${ }^{12} \mathrm{C}-{ }^{12} \mathrm{C}$ scatterings are shown in Figs. 1 and 2 together with the results of the microscopic coupled-channels calculation of ${ }^{16} \mathrm{O}-{ }^{16} \mathrm{O}$ and ${ }^{12} \mathrm{C}-{ }^{12} \mathrm{C}$ scatterings published in the original paper. On the whole, the $3 \mathrm{NF}$ effects persist, improving the correspondence between the calculated and measured cross sections, although the agreement is insufficient at large angles. It has been confirmed that the $3 \mathrm{NF}$ effects on the total reaction cross sections are still small. Our conclusion for the $3 \mathrm{NF}$ effects in the nucleus-nucleus elastic and inelastic scatterings does not change qualitatively.

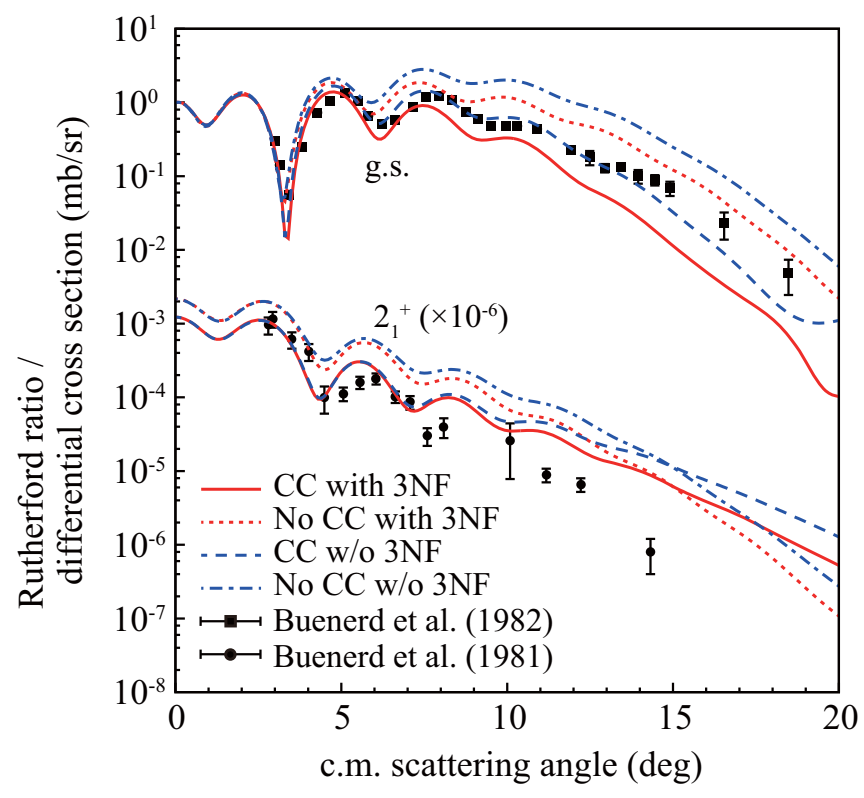

FIG. 2. Revised version of Fig. 2(a) in the original paper. Differential cross sections of elastic and inelastic ${ }^{12} \mathrm{C}-{ }^{12} \mathrm{C}$ scatterings at $85 \mathrm{MeV} /$ nucleon. The meaning of the lines is the same as in Fig. 1. The experimental data are taken from Refs. [5,6].
[1] M. Kohno, this issue, Phys. Rev. C 96, 059903 (2017).

[2] M. Kohno, Phys. Rev. C 88, 064005 (2013).

[3] F. Nuoffer et al., Nuovo Cimento A 111, 971 (1998).

[4] D. T. Khoa et al., Nucl. Phys. A759, 3 (2005).

[5] M. Buenerd et al., Phys. Lett. B 102, 242 (1981).
[6] M. Buenerd, P. Martin, R. Bertholet, C. Guet, M. Maurel, J. Mougey, H. Nifenecker, J. Pinston, P. Perrin, F. Schussler, J. Julien, J. P. Bondorf, L. Carlen, H. A. Gustafsson, B. Jakobsson, T. Johansson, P. Kristiansson, O. B. Nielsen, A. Oskarsson, I. Otterlund, H. Ryde, B. Schroder, and G. Tibell, Phys. Rev. C 26, 1299 (1982). 